\title{
The Evaluation of the V2VUNet Concept to Improve Inter-vehicle Communications
}

\author{
Lisa Kristiana ${ }^{1,2(\mathbb{})}$, Corinna Schmitt ${ }^{1}$, and Burkhard Stiller ${ }^{1}$ \\ ${ }^{1}$ Communication Systems Group CSG, Department of Informatics IfI, \\ University of Zürich, Binzmühlestrasse 14, 8050 Zurich, Switzerland \\ \{kristiana, schmitt, stiller\}@ifi.uzh.ch \\ 2 The Department of Informatics, National Institute of Technology, \\ Bandung 40124, Indonesia \\ lisa@itenas.ac.id
}

\begin{abstract}
Due to the high mobility behavior in inter-vehicle communications (IVC), packet forwarding among vehicles becomes an important issue. For IVC in a traditional packet forwarding setting, it was observed that the ratio between packets received and the packets transmitted is often very low, sometimes less than $50 \%$. This ratio is highly influenced by the environment, especially by road topologies and obstructions (e.g., buildings or overpasses). Further influences encompass the number of driving vehicles on streets offering burdens for the IVC as well as serving as relay candidates. In order to improve IVC this paper introduces a Vehicular-to-Vehicular Urban Network (V2VUNet) to overcome inevitable obstructions and frequent network changes by selecting the best relay candidate. The V2VUNet implemented was evaluated in an IVC with the focus on three-dimensional road topologies including overpasses with a different number of driving lanes. The result shows that the developed V2VUNet provides about $30 \%$ better packet transmission performance compared to traditional packet transmission in IVC.
\end{abstract}

\section{Introduction}

Inter-vehicle networks as a part of Vehicular Ad-hoc Network (VANET) are expected to support communications with multiple participating vehicles [3]. Thus, information exchanges in a vehicular network communication require stable and reliable connections. During packet transmissions and receptions the communication path has to be maintained in any cases. For IVC in a traditional packet forwarding setting, it was observed that the Packet Delivery Ratio (PDR) as the indicator of network performance is often less than $50 \%$ due to path failures [19]. These path failures are mainly caused by the road topology complexity of the environment, such as overpass constructions and buildings at intersection roads [9].

For inter-vehicle communications, a position-based forwarding scheme is generally used, since it offers an advantage of not relying on packet broadcasting in its routing mechanisms. Since position information is already made available, the approach proposed in this paper, the Vehicular-to-vehicular Urban Network (V2VUNet), emphasizes in filtering unnecessary participant nodes [18] and predicting the routing path based on 
the position information and on the calculated direction information [17]. Therefore, the packet forwarding scheme is expected to become more efficient. In this research work, the packet forwarding is tested in two types of road environment models. The first type is the cross road model and the second type is the parallel road model. Both types reflect the three-dimensions cases with three coordinate axes $x, y$, and $z$. The advance beyond state-of-the-art in this three-dimensional area is determined by the $z$ axis, which in many of VANET scenarios is rarely included. In addition, the three-dimensional case in VANET is significantly influenced by objects placed between signal transmitting devices. These objects can be a building or an overpass. Therefore, it is important to investigate the packet forwarding in these three-dimensional environments. Besides a better performance, the packet forwarding scales best in case of non-safety or non-real-time applications, which can be considered as delay constraint. Thus, delays are evaluated here as a less significant characteristic.

Another relevant aspect today is the use of Multiple Input Multiple Output (MIMO) technology in Inter-vehicle Communication (IVC). MIMO is a method to increase the radio link capacity and becomes a promising solution, since it increases the number of transmitted data by embedding multiple transmitters and receivers [15]. This method is useful to be implemented in a non-safety application, such as for infotainment, since it requires high data rates and large amount of data interchanges. However, as this work in this paper here focuses on the network layer, MIMO is better to be evaluated in the data link layer.

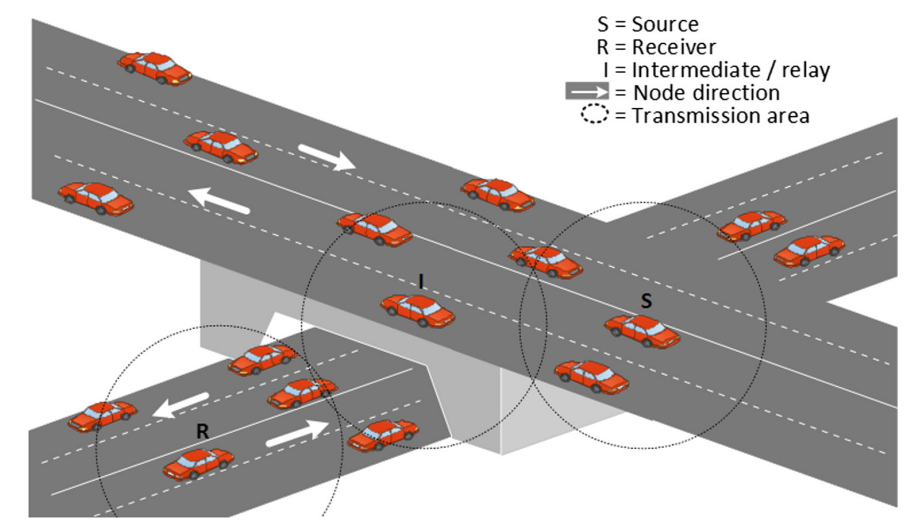

Fig. 1. Inter vehicle communication in a three-dimensional road topology

In previous works as shown in $[17,18]$, the evaluation of network performance of each approach is compared to a traditional location-based routing. This paper ensues to evaluate both forwarding approaches in V2VUNets by implementing various size of packets, different network densities, and speed of vehicles. The remainder of this paper is organized as follows: Sect. 2 describes related work of the packet forwarding model used. Section 3 introduces the key idea of the vertical angle forwarding scheme being part of a V2VUNet. Additionally, the evaluation of the V2VUNet is discussed in Sect. 4 , followed by the summary and future work in Sect. 5 . 


\section{Related Work}

Successful communication requires an efficient packet forwarding. Packet forwarding is considered as efficient, when the packet is broadcasted with a smaller probability of errors. Packet forwarding for non-safety applications refers to numerous size of data and is assumed to be distributed at a high rate [1-3]. Therefore, dealing with frequent topology changes in IVC's behavior, the packet forwarding is based on the method of forwarding [2, 5-7]. The first idea is to avoid collision in a dense network [8], thus, the packet forwarding is designed to reduce the number of relay candidates by restricting the area of transmission [18]. The second idea is to predict the direction of relay candidates by calculating the relative direction of a relay and by selecting the candidate that has the same direction with the destination's direction [17]. It is obvious that the relay having the same direction with the destination increases the possibility to prolong the duration of a connection between communicating vehicles. Thus, in a large city environment with its road topology and traffic complexity [5], both approaches will be evaluated to perform a reliable data transmission in VANETs, more specifically in IVC. The two concepts of packet forwarding in IVC are studied in a survey that shows relevant literatures $[4-7,16]$. While the first concept follows an angle-based forwarding approach, the second concept is defined as direction-based forwarding approach.

\subsection{Angle-Based Forwarding}

An angle-based forwarding mechanism utilizes angle measurement to reduce the area of transmission. The idea of implementing angle is to locate relay candidates within the transmission range of a sender $S$. Thus, under the assumption that the location coordinate of a receiver $R$ is known, the imaginary line is drawn in order to scale the angle as shown in Fig. 2. The angle-based forwarding mechanism selects one of the relay candidates as the intermediate relay based on the location where it has the smallest angle calculation respect to an imaginary line [11, 12]. As illustrated in Fig. 2, $S$ selects node $C$ as the relay node since $C$ has the smallest angle value. The advantage of this mechanism suits on the dense network because of the efficient route path in terms of time $[12,13]$. The comparison of existing angle forwarding schemes has been done in [21].

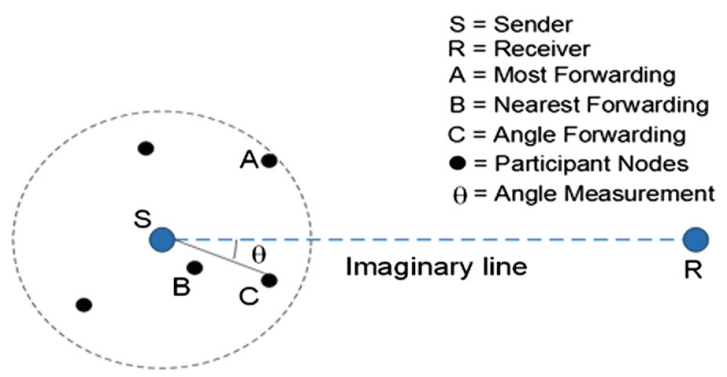

Fig. 2. Angle forwarding scheme 


\subsection{Direction-Based Forwarding}

In IVC, vehicles are assumed to move on a predefined path such a straight or intersection road. Thus, vehicles can have heterogeneous directions depend on the road types. In order to cover route loss due to the 'free' movement of vehicles, the direction-based forwarding mechanism involves direction as a weight value to determine the next relay node. The direction has a dynamic value since it depends on the road topology and time-based factor. The direction value is calculated based on the sender and receiver position as illustrated in Fig. 3.

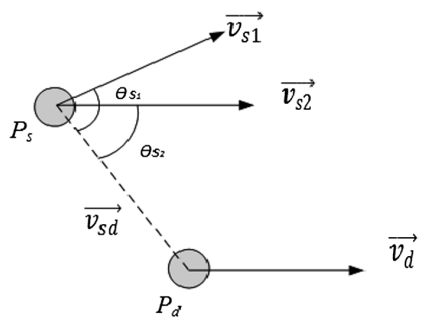

Fig. 3. A relative direction of a vehicle

\section{V2VUNet Concept}

The concept used in V2VUNet is an enhancement of the selection method in order to find the best relay node of available candidates [1, 4]. Previous work of V2VUNet determines the area restriction of transmission [18] and the path prediction [17]. Both area restriction and path prediction schemes in V2VUNet utilize the angle measurement on the same road level, i.e., Horizontal Relative Angle (HRA) measurement and different road levels i.e., Vertical Relative Angle (VRA) measurement. The implementation of V2VUNet in this work is performed to compare each algorithm in various parameters.

\subsection{Area Restriction Scheme}

In the area restriction scheme, the V2VUNet operates in two steps. The first step is to define the HRA with value of $30^{\circ}$, which is intuitively based on the width of a road in two-dimensional area and the closest distance between two vehicles. The second step is to adjust HRA based on the available relay candidates position. If the number of relay candidates is more than one, then the V2VUNet algorithm selects the relay based on the smallest value of HRA. The algorithm for the area restriction scheme is shown as in Algorithm 1 (cf. Fig. 4). The previously mentioned two steps are also applied in a three-dimensional area. Similar to HRA, VRA will be first defined as $30^{\circ}$, which is indicated as the preliminary angle value based on the transmission range. This $30^{\circ}$ value is then increased gradually as part of the searching mechanism. 
Algorithm 1
1. $S \leftarrow$ sender node
2. $R \leftarrow$ receiver node
3. / all neighboring nodes of $S$
4. $\theta_{x, \text { max }}+$ maximum boundary of the horizontal angle
5. $\theta_{z, \min } \leftarrow$ minimum boundary of the vertical angle
6. $\theta_{z, \max } \leftarrow$ maximum boundary of the vertical angle
7. $\theta_{x} \leftarrow$ horizontal angle made by $n$ to $s$
8. $\theta_{z}+$ vertical angle made by $n$ to $s$
9. $i_{\text {fittered }} \leftarrow$ only $i$ that is within $\left[-\theta_{x, \max }, \theta_{x, \max }\right]$ and $\left[-\theta_{z, \min }, \theta_{z, \max }\right]$
10. $d$ + distance from ifiltered to $R$, nexthop $\leftarrow \arg _{\min }(d)$

Fig. 4. Area restriction algorithm

\subsection{Path Prediction Scheme}

In this scheme, the HRA is used to predict the direction of relay candidates. The prediction algorithm is designed to overcome the disconnection possibility due to the transmission coverage in two-dimensional area. In case of VRA, the algorithm is designed to encounter the disconnection due to obstruction by the overpass. Furthermore, HRA and VRA in this scheme use the relative direction of each vehicle. This relative direction provides the actual direction in three-dimensional scheme. The path prediction scheme is shown in Algorithm 2 (cf. Fig. 5).

Algorithm 2

1. $s+$ sender node, at position of $p_{s}$ and orientation of $v_{s}$

2. $i$ all neighboring nodes of $s$, at position of $p_{i}$ and orientation of $v_{i}$

3. $\theta_{\text {solid }}+$ threshold of the solid angle for all $i$

4. $v_{s i}=\left|p_{s}-p_{i}\right|$

5. $\theta_{s i}=\operatorname{atan}\left(\left\|v_{s} \times v_{s j}\right\|,\left\|v_{s} \cdot v_{s i}\right\|\right)$

6. initered $+i$ with $\theta_{s i}$ within [- $\left.\theta_{\text {Solidi }} \theta_{\text {solid }}\right]$

7. $d$ \& distance from $i_{\text {filtered }}$ to $R$

8. next hop $+\operatorname{argmin}(d)$

Fig. 5. Path prediction algorithm

For the sake of a precise prediction, the direction that is used in V2VUNet algorithm determines a relative direction since the direction of each vehicle is measured in vehicle's current position. This relative direction is changed whenever a vehicle changes its position as illustrated in Fig. 3. Thus, the traditional direction calculation cannot be implemented in this prediction as it is done in [17]. Those two proposed algorithms are expected to provide a network performance as indicated by high PDR and low end-to-end (E2E) delays. In order to compare all algorithms implemented in this work, Table 1 shows a short description of each scheme and all related factors. 
Table 1. Comparison of angle, area restricted, and path prediction forwarding algorithms

\begin{tabular}{l|l|l|l}
\hline Factor & $\begin{array}{l}\text { Angle-based } \\
\text { forwarding }\end{array}$ & Area restriction & Path prediction \\
\hline $\begin{array}{l}\text { Coordinate } \\
\text { location }\end{array}$ & $x$-, and $y$-axis & $x$-, $y$-, and $z$-axis & $x$-, y-, and $z$-axis \\
\hline Weight value & HRA & HRA and VRA & HRA and VRA \\
\hline Relative direction & No & No & Yes \\
\hline Routing based & Greedy & Greedy & Greedy \\
\hline Road topology & $\begin{array}{l}\text { 2D intersection, } \\
\text { highway }\end{array}$ & $\begin{array}{l}\text { 3D intersection, 3D } \\
\text { parallel }\end{array}$ & $\begin{array}{l}\text { 3D intersection, 3D } \\
\text { parallel }\end{array}$ \\
\hline
\end{tabular}

As the first factor in this comparison table the coordinate location describes the coordinate axis which is used in measuring the current location of a node. The second factor is the weight value which determines the angle schemes, HRA for two-dimensional area and VRA for three-dimensional area. The third factor is the relative direction which is added in the path prediction algorithm in order to improve the calculation of the location coordinate. This relative direction factor is suitable when nodes move randomly and is useful to indicate the current direction of a node. Greedy routing is used in all algorithms because greedy routing uses the distance factor to do packet forwarding. Basically the greedy approach work best the many routing protocol mechanisms [10]. The last factor that influences all forwarding schemes is the road topology, which becomes the main idea of forwarding packet improvement. The angle-based forwarding scheme is used in two-dimensional intersection, where the direction factor becomes an important value, and in the highway, where the speed of a vehicle is highly considered. However, it is necessary to consider about the complexity of a road topology. Thus, the area restriction and path prediction schemes raise the three-dimensional road topology indicated as three-dimensional intersection (i.e., cross road) and parallel roads.

As previously mentioned in the introduction section, this work evaluates and compares the area restriction and path prediction schemes as the improvement of traditional greedy routing.

\section{Performance and Evaluation}

The simulation in this work aims to validate the theoretical analysis of the proposed algorithms in IVC. Two simulation scenarios of a road environment with parallel and cross road topology are selected. In parallel road topology, the difference in vehicles' direction is more extreme than in a cross road topology. In a parallel topology, there are less chance that one vehicle can meet another vehicle once they pass each other. In a cross road topology, there is a segment of the road that is under another segment of the road, which could potentially contributes to disconnection at particular moment.

In order to obtain a realistic city environment, typical parameters for the influencing factors are chosen as shown in Table 2. The Network Simulator-3 (NS-3.25) [14] is used to simulate wireless technologies (i.e., IEEE 802.11p), the routing protocol (i.e., Greedy Perimeter Source Routing (GPSR) [10]), the mobility, the road topology, and 
the network density. The IEEE 802.11p is a well known technology since it is designed to cope the frequent topology changing in IVC. During $200 \mathrm{~s}$ of simulation time, each vehicle is expected to run under and on the overpass in the first case, and on the different road level in the second case. $S, R$, and $I$ are placed randomly both on two different road levels and SUMO [20] is used to generate the realistic mobility of each vehicle. Moreover, the number of $S$ and $R$ are generated equally, which means a 10 -vehicle network contains of 5 senders and 5 receivers. The simulation area covers an environment which involves crossing and parallel overpass scenarios (c.f. Fig. 1) in order to show many cases in three-dimensional area.

In previous works, these two algorithms have not been evaluated over various packet sizes, thus, the packet size for the first evaluation is varied from $1-10 \mathrm{kB}$, especially for non-safety applications: a half page of unformatted email is $1 \mathrm{kB}$, one typical HTML webpage is $30 \mathrm{kB}, 1$ min of near-CD quality audio as MP3 or a $2048 \times 1536$ (4 megapixel) JPEG photo is $1 \mathrm{MB}$, to evaluate the size of packet that can be successfully transmitted in two algorithms. However, this simulation focuses only on transmitting email, with the size of the packet from 1 to $10 \mathrm{kB}$.

The first result (cf. Fig. 6) shows the PDR of all algorithms when different packet sizes are applied. This performed evaluation simulates 40 vehicles that move with an average speed of $40 \mathrm{~km} / \mathrm{h}$. The V2VUNet area restriction algorithm gives $20 \%$ better PDR compared to the greedy forwarding scheme which in the figure is indicated as No V2VUNet. V2VUNet area restriction also shows $10 \%$ better compared to V2VUNet-path prediction scheme. The showed PDR in overall algorithms decreases as the packet size increases, which indicates that more participating vehicles and simulation time are required to successfully complete the packet forwarding mechanism. V2VUNet indicates that HRA and VRA weight values have significant impacts in packet transmission.

Table 2. Parameter Settings

\begin{tabular}{l|l}
\hline Parameter & Unit \\
\hline Transmission range IEEE 802.11p & Up to $300 \mathrm{~m}$ \\
\hline Routing protocols & GPSR \\
\hline Number of vehicles & $20-100$ \\
\hline Simulation area & $500 \mathrm{~m} \times 500 \mathrm{~m}$ \\
\hline Upper road height & $20 \mathrm{~m}$ \\
\hline Vehicle velocity & $30-70 \mathrm{~km} / \mathrm{h}$ \\
\hline Packet size & $1 \mathrm{kB}-10 \mathrm{kB}$ \\
\hline Simulation time & $200 \mathrm{~s}$ \\
\hline Number of driving lanes & 4 \\
\hline DSRC data rates & $6 \mathrm{Mbps}$ \\
\hline
\end{tabular}

The second result shows the E2E delay of all algorithms (cf. Fig. 7) when different data sizes are applied. The E2E delay increases as the packet size increases. This is because more time are required to transmit packet with bigger size. The E2E delay of $200 \mathrm{~ms}$ (highest delay) is indicated by path prediction scheme. This is because the path finding mechanism in the V2VUNet path prediction scheme requires more time 


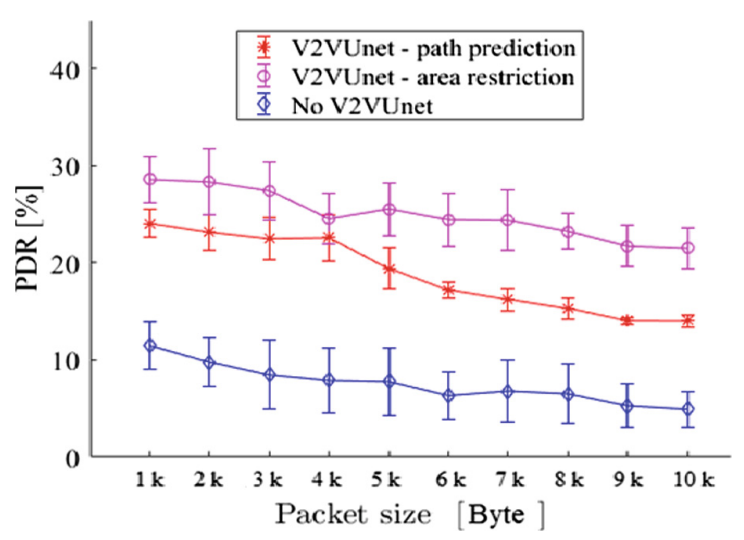

Fig. 6. Evaluation of a packet delivery ratio

compared to the area restriction mechanism. In overall E2E delay results, the traditional forwarding scheme provides lowest delay compared to other two algorithms. This is because the traditional forwarding scheme does not need an additional mechanism to perform packet forwarding.

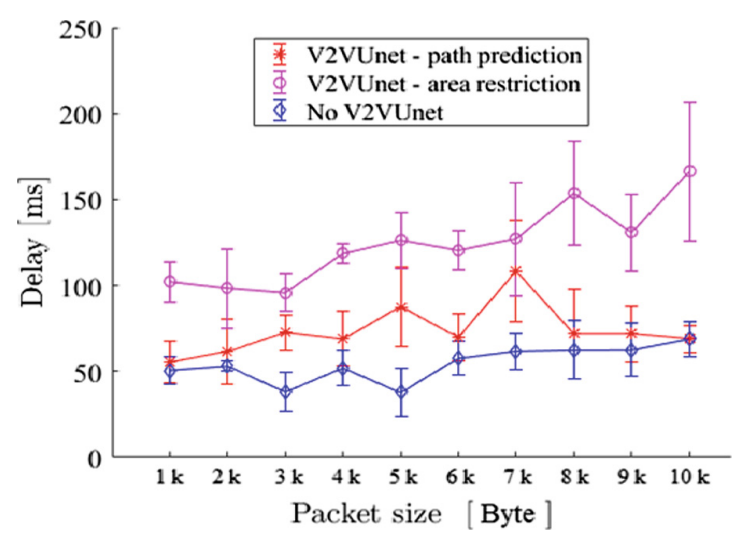

Fig. 7. Evaluation of end-to-end delays

The third set of results shows PDR of all algorithms in various number of participating vehicles in cross road scenario (cf. Fig. 8). These various number of vehicles are used to evaluate V2VUNet schemes when dealing with the network density and network speed. The V2VUNet-path prediction and V2VUNet-area restriction shows $10 \%$ and $20 \%$ better PDR, respectively, compared to a greedy forwarding without V2VUNet. As the network density grows, the PDR decreases in all forwarding schemes. From all simulation trials that have been performed, the highest PDR that can be achieved is about $40 \%$. This is due to the path failure that occurs when the communicating vehicles are under the overpass. This path failure cannot be avoided since 

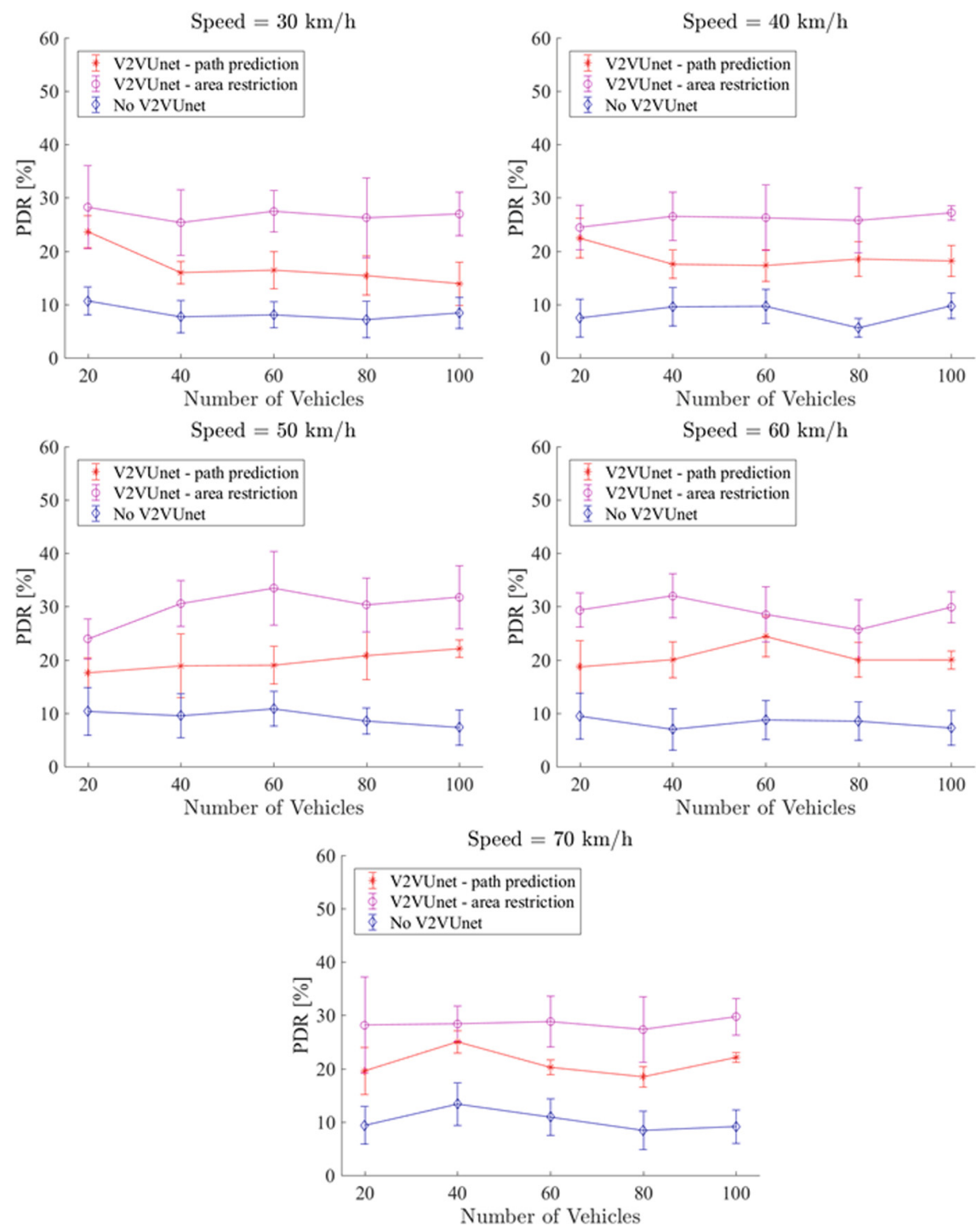

Fig. 8. Evaluation of packet delivery ratios for varying numbers of vehicles and vehicle speeds

the overpass disturbs the transmission, thus it will never reach $100 \%$ of PDR. The network density with 60-80 vehicles shows the maximum $40 \%$ result at speed $50 \mathrm{~km} / \mathrm{h}$. This indicates that 60 vehicles with 30 pairs of $S$ and $R$ are the 'best' condition where the packet transmission is performed.

However, in high speed mobility (i.e. $50 \mathrm{~km} / \mathrm{h}-70 \mathrm{~km} / \mathrm{h}$ ), the PDR reaches higher results compared to PDR in low speed mobility (i.e., $30 \mathrm{~km} / \mathrm{h}$ and $40 \mathrm{~km} / \mathrm{h}$ ). The main reason for this is that in higher speed mobility, the path reconstruction is even more possible than maintaining the old path. In this case, V2VUNet area restriction scheme 
provides PDR 10\% higher compared to V2VUNet path prediction forwarding scheme. This is caused by the overpass construction which blocks the packet transmission, thus, it becomes difficult to complete path finding process.

The fourth set of results shows E2E delays of all algorithms when various number of participating vehicles are involved in a cross road scenario (cf. Fig. 9). This E2E delay reaches $350 \mathrm{~ms}$ at $70 \mathrm{~km} / \mathrm{h}$ speed. The high E2E delay can be caused by two reasons: the first reason is that the intermediate node which moves in the opposite direction (e.g., vehicle that changes its direction or turns back), has impact to the searching mechanism. Thus, the mechanism starts to find a new path and the transmission is delayed because of this reason. The second reason is that the connections between two vehicles are interrupted or discontinued, when one of the vehicles is located under the overpass. However, the E2E delays decrease for mobility with higher speeds i.e. $50 \mathrm{~km} / \mathrm{h}-70 \mathrm{~km} / \mathrm{h}$. The similar explanation as in the PDR can also be applied to explain the E2E delays. The required period of time to find a new path is less than in the mobility with low speeds (i.e., $30 \mathrm{~km} / \mathrm{h}$ and $40 \mathrm{~km} / \mathrm{h}$ ). In overall results, the
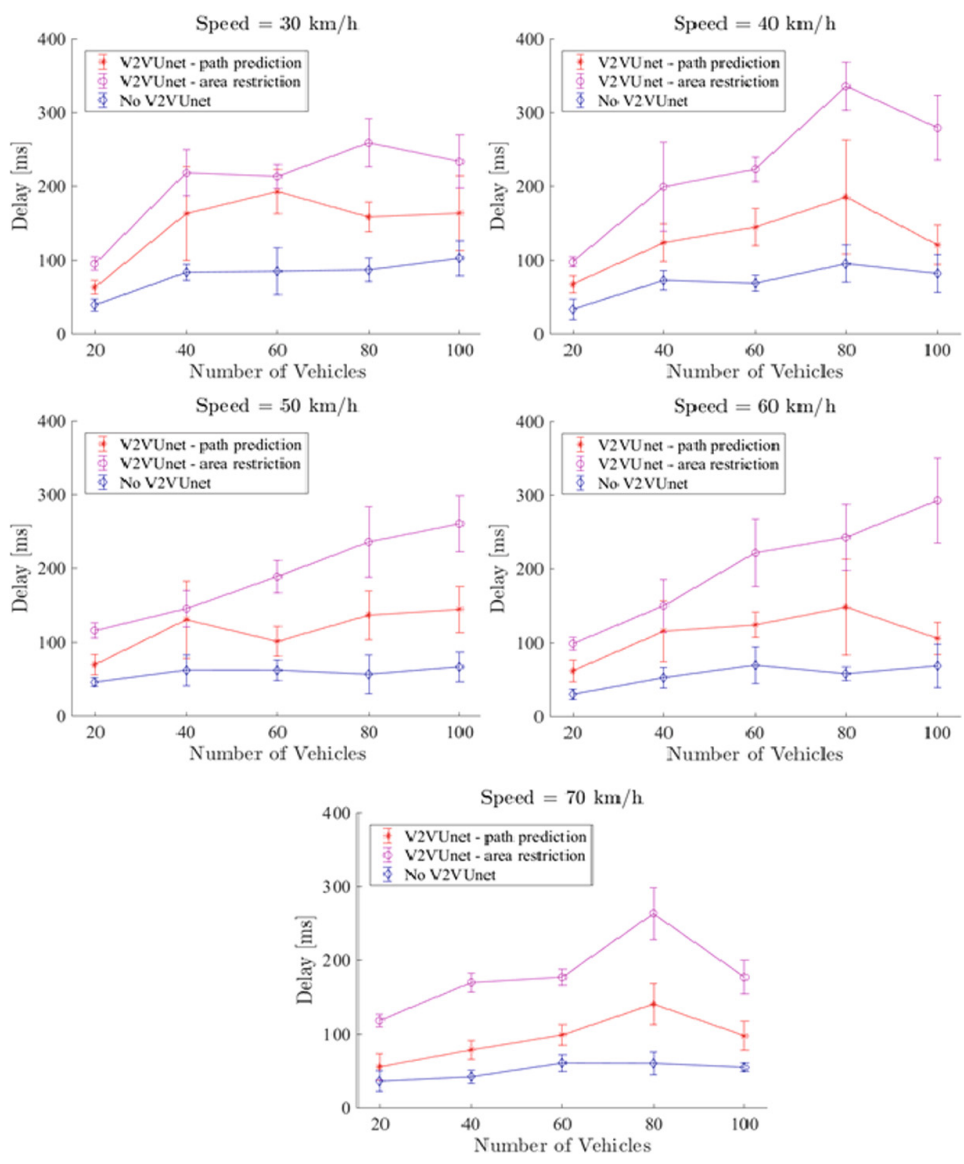

Fig. 9. Evaluation of end-to-end delays for varying numbers of vehicles and vehicle speeds 

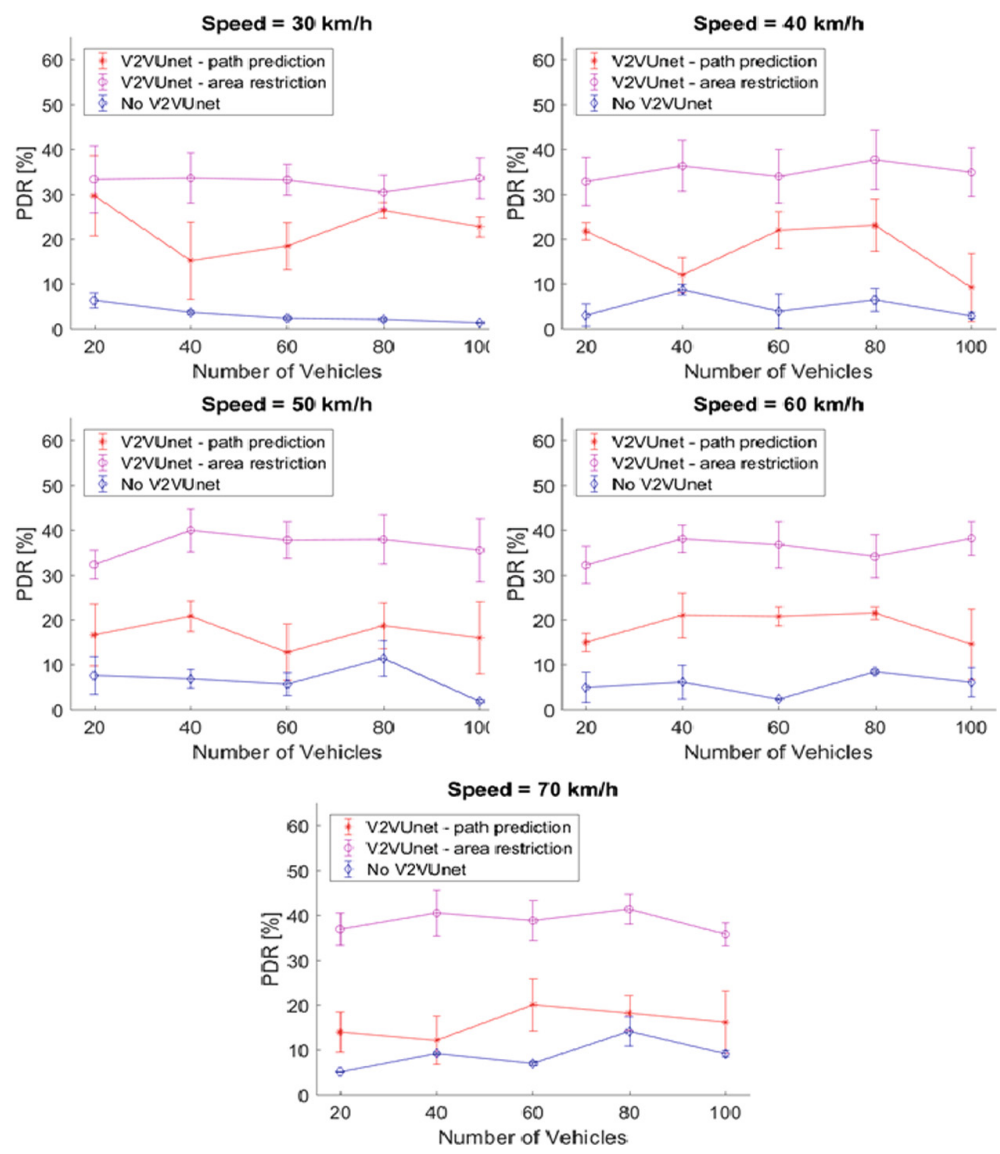

Fig. 10. Evaluation of packet delivery ratios for varying numbers of vehicles and vehicle speeds

E2E delays are considered as drawbacks in order to obtain better PDR by applying V2VUNet. Thus, this becomes an open question.

The fifth set of results in Fig. 10 shows the evaluation of PDR in the parallel road scenario. Here, the path prediction scheme reaches $40 \%$. When compared to other schemes, PDR of the path prediction is found to be the highest because in parallel road scenarios the direction of vehicles is predictable i.e., either in the same direction or opposite direction. Thus, in the parallel scenario, path prediction scheme works well in predicting the relay candidate's direction. In overall, the PDR decreases accordingly to number of participating nodes. This is because of the collision due to the network density.

The sixth set of results shows E2E delays (cf. Fig. 11) in the parallel road scenario. The traditional greedy routing shows the lowest delay compared to other scheme because the scheme does not include additional searching mechanism as previously mentioned. However, the path prediction scheme shows reasonable E2E delays of $50 \mathrm{~ms}$ as the prediction mechanism works well in the parallel road scenario. 

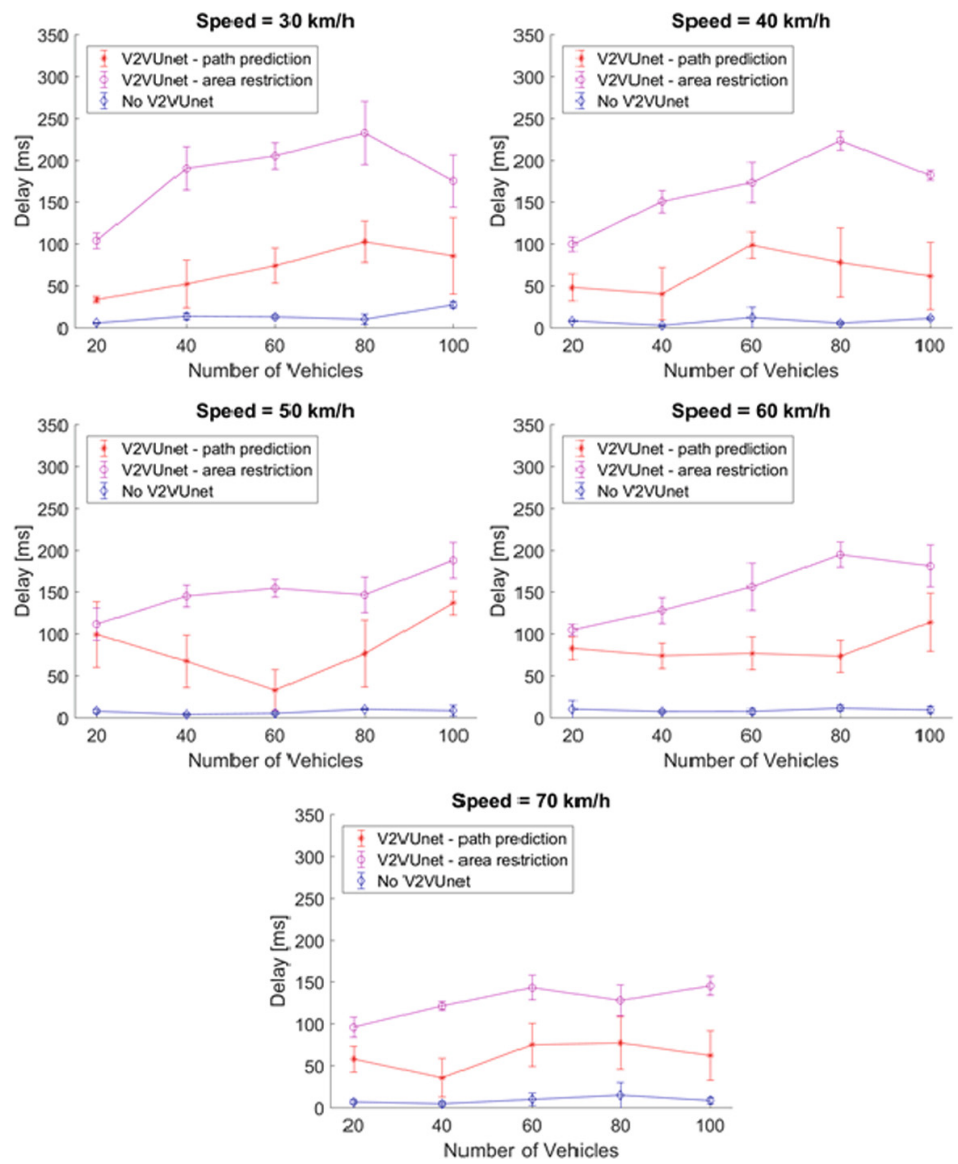

Fig. 11. Evaluation of end-to-end delays for varying numbers of vehicles and vehicle speeds

\section{Summary and Future Work}

This work covers and ensues the evaluation of V2VUNet through a three-dimensional road topology in a large city. Important parameters of V2VUNet have been evaluated: packet size, speed, and number of vehicles. The V2VUNet takes into account HRA and VRA as additional weight values, which are applied in area restriction and path prediction algorithms. The network performance as indicated by PDR and E2E delay values shows to be reliable in non-safety applications. The PDR in an overall performance shows that V2VUNet provides $20 \%$ better result compared to traditional routing algorithms. However, the E2E delays in the overall evaluations are slightly higher than for traditional routing algorithms. Thus, these E2E delays are considered to determine the trade-off in V2VUNet, even though a non-safety application is assumed to be a delay tolerant scheme. Additionally, the path prediction scheme is less suitable to be adopted in the cross scenario, however, it performs better in the parallel scenario. Thus, 
it can be concluded that the V2VUNet path prediction works better in the parallel scenario, since the direction of vehicles is homogeneous. The V2VUNet area restriction performs better in the cross scenario, since it restricts the number of relay candidates.

Further research in improving V2VUNet, the area restriction, and path prediction concept can be performed for any position-based routing scheme, where the distance and direction indicate the influencing weight value. The combination of these two schemes will be considered as a hybrid scheme, thus, both algorithms in V2VUNet are expected to improve the packet forwarding scheme depending on the use case.

\section{References}

1. Mauve, M., Scheuermann, B.: VANET Convenience and Efficiency Applications, VANET Vehicular Applications and Inter-Networking Technology, pp. 81-105. Wiley, Hoboken (2010)

2. Aoki, M., Fujii, H.: Inter-vehicle communication: technical issues on vehicle control application. IEEE Commun. Mag. 34(10), 90-93 (2002). New York, NY, U.S.A. doi:10. $1109 / 35.544327$

3. Papadimitratos, P., La Fortelle, A.D., Paristech, M., Evenssen, K., Asa, Q.: Vehicular communication systems: enabling technologies, applications, and future outlook on intelligent transportation. IEEE Commun. Mag. 47(11), 84-95 (2009). New York, NY, U. S.A. doi:10.1109/MCOM.2009.5307471

4. Jerbi, M., Senouci, S.M., Meraihi, R., Ghamri-Doudane, Y.: An improved vehicular ad-hoc routing protocol for city environments. In: IEEE International Conference on Communications, New York, N.Y, U.S.A., pp. 3972-3979, June 2007. doi:10.1109/ICC.2007.654

5. Tavakoli, R., Nabi, M.: TIGeR: a traffic-aware intersection-based geographical routing protocol for urban VANETs. In: IEEE 77th Vehicular Technology Conference (VTC Spring), New York, N.Y., U.S.A., pp. 1-5, June 2013. doi:10.1109/VTCSpring.2013. 6692786

6. Lin, Q., Li, C., Wang, X., Zu, L.: A three-dimensional scenario oriented routing protocol in vehicular ad-hoc networks. In: IEEE Vehicular Technology Conference (VTC), New York, N.Y., U.S.A., pp. 1-5, June 2013. doi:10.1109/VTCSpring.2013.6691848

7. Wang, H., Tan, G.Z., Yang, J.X.: An improved VANET intelligent forward decision-making routing algorithm. J. Netw. 7(10), 1546-1553 (2012)

8. Mohimani, G.H., Ashtiani, F., Javanmard, A., Hamdi, M.: Mobility modeling, spatial traffic distribution, and probability of connectivity for sparse and dense vehicular ad-hoc networks. IEEE Trans. Vehicular Technol. 58(4), 1998-2007 (2009). New York, N.Y., U.S.A. doi:10. 1109/TVT.2008.2004266

9. Walfisch, J., Bertoni, H.: Theoretical model of UHF propagation in urban environments. IEEE Trans. Antennas Propag. 36(12), 1788-1796 (1988). New York, N.Y., U.S.A. doi:10. $1109 / 8.14401$

10. Karp, B., Kung, H.T.: GPSR: greedy perimeter stateless routing for wireless network. In: International Conference on Mobile Computing and Networking (MobiCom), pp. 243-254. ACM, New York, August 2000. doi:10.1145/345910.345953

11. Spachos, P., Toumpakaris, D., Hatzinakos, D.: Angle-based dynamic routing scheme for source location privacy in wireless sensor network. In: IEEE Vehicular Technology Conference (VTC), New York, N.Y., U.S.A., pp. 1-5, May 2014. doi:10.1109/VTC-Spring. 2014.7022833 
12. Banka, R.K., Xue, G.: Angle routing protocol: location aided routing for mobile ad-hoc networks using dynamic angle selection. In: IEEE Premier International Conference for Military Communications (MILCOM), New York, N.Y., U.S.A., vol. 1, pp. 501-506, October 2001. doi:10.1109/MILCOM.2002.1180493

13. Huang, C., Chiu, Y., Wen, C.: Using hybrid angle/distance information for distributed topology control in vehicular sensor networks. Sensors 14(11), 20188-20216 (2014). Basel, Switzerland, doi:10.3390/s141120188

14. NS3. https://www.nsnam.org/. Accessed 28 Jan 2017

15. Moser, S., Behrendt, L., Slomka, F.: MIMO-enabling PHY layer enhancement for vehicular ad-hoc networks. In: IEEE Wireless Communications and Networking Conference Workshops (WCNCW), New York, N.Y., U.S.A., pp. 142-14 (2015). doi:10.1109/WCNCW.2015.7122544

16. Kristiana, L., Schmitt, C., Stiller, B.: Survey of angle-based forwarding methods in VANET communications. In: IEEE Wireless Days (WD), Toulouse, France, pp. 1-3, March 2016. doi:10.1109/WD.2016.7461505

17. Kristiana, L., Schmitt, C., Stiller, B.: Predictive forwarding scheme in three-dimensional vehicular communication scenarios. In: International Conference on Selected Topics in Mobile and Wireless Networking (MoWNeT), Avignon, France, pp. 1-6, May 2017

18. Kristiana, L., Schmitt, C., Stiller, B.: V2VUNet - a filtering out concept for packet forwarding decision in three-dimensional inter-vehicular communication scenarios. In: IEEE 27th Annual International Symposium on Personal, Indoor, and Mobile Radio Communications (PIMRC), New York, N.Y., U.S.A., pp. 1-6, September 2016. doi:10.1109/PIM-RC. 2016.7794600

19. Kolici, V., Oda, T., Spaho, E., Barolli, L., Ikeda, M., Uchida, K.: Performance evaluation of a VANET simulation system using NS-3 and SUMO. In: IEEE 29th International Conference on Advanced Information Networking and Applications Workshops (WAINA), New York, N.Y., U.S.A., pp. 348-353, March 2015. doi:10.1109/WAINA.2015.121

20. Simulation of Urban MObility, SUMO. http://www.dlr.de/ts/en/desktopdefault.aspx/tabid9883/16931_read-41000/. Accessed 16 Mar 2017

21. Kristiana, L., Schmitt, C., Stiller, B.: Survey of angle-based forwarding methods in VANET communications. In: IEEE Wireless Days, New York, N.Y., U.S.A., pp. 1-3, March 2016. https://doi.org/10.1109/WD.2016.7461505

Open Access This chapter is licensed under the terms of the Creative Commons Attribution 4.0 International License (http://creativecommons.org/licenses/by/4.0/), which permits use, sharing, adaptation, distribution and reproduction in any medium or format, as long as you give appropriate credit to the original author(s) and the source, provide a link to the Creative Commons license and indicate if changes were made.

The images or other third party material in this chapter are included in the chapter's Creative Commons license, unless indicated otherwise in a credit line to the material. If material is not included in the chapter's Creative Commons license and your intended use is not permitted by statutory regulation or exceeds the permitted use, you will need to obtain permission directly from the copyright holder.

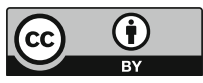

